Pacific

Journal of

Mathematics

ON THE HAUSDORFF $h$-MEASURE OF CANTOR SETS

Carlos Cabrelli, Franklin Mendivil, Ursula M. Molter AND RONALD SHONKWILER 


\title{
ON THE HAUSDORFF $h$-MEASURE OF CANTOR SETS
}

\author{
Carlos Cabrelli, Franklin Mendivil, Ursula M. Molter \\ AND RONALD SHONKWILER
}

\begin{abstract}
We estimate the Hausdorff measure and dimension of Cantor sets in terms of a sequence given by the lengths of the bounded complementary intervals. The results provide the relation between the decay rate of this sequence and the dimension of the associated Cantor set.

It is well-known that not every Cantor set on the line is an $s$-set for some $0 \leq s \leq 1$. However, if the sequence associated to the Cantor set $C$ is nonincreasing, we show that $C$ is an $h$-set for some continuous, concave dimension function $h$. We construct the function $h$ from the sequence associated to the set $C$.
\end{abstract}

\section{Introduction}

A Cantor set is a compact, perfect, totally disconnected subset of the real line. In this article we will consider only Cantor sets of Lebesgue measure zero. The complement of a Cantor set is a countable union of disjoint open intervals. We will use the term gap for any bounded convex component of the complement of a Cantor set.

Every Cantor set is completely determined by its gaps. Since the gaps are disjoint, the sum of their lengths equals the diameter of the Cantor set.

There is a natural way to associate to each summable sequence of positive numbers a unique Cantor set having gaps with lengths equal to the terms of the sequence. In this correspondence the order of the sequence is important. Different rearrangements can lead to different Cantor sets. Of course, if two sequences lead to the same Cantor set, one is a rearrangement of the other.

In the first part of this paper we will concentrate on finding the Hausdorff measure of a Cantor set in terms of the decay of the sequence of the lengths of the gaps. In particular we will show that the Hausdorff dimension depends totally on this behavior.

We establish an equivalence relation between sequences and show that Cantor sets in the same equivalence class have the same dimension.

Since the Cantor set depends on the order of the sequence, one expects that the dimension of the resulting set also depends on the order. This 
is true, and moreover, the arrangement of the sequence in monotone nonincreasing order yields the Cantor set with the largest dimension out of all Cantor sets with the same set of gap lengths (see also [BT54]).

Let $0 \leq s \leq 1$. An $s$-set is a set on the line of Hausdorff dimension $s$ and whose Hausdorff $s$-measure is finite and positive. Let $h$ be a nondecreasing, right-continuous function taking the value zero at the origin. The Hausdorff $h$-measure $\mathcal{H}^{h}$ is defined in the same way as the Hausdorff $s$-measure but replaces the function $x^{s}$ by $h(x)$ (see [Rog98], [Hau19]):

$$
\mathcal{H}^{h}(A)=\lim _{\delta \rightarrow 0} \inf \left\{\sum h\left(\operatorname{diam} E_{i}\right): E_{i} \text { open, } \bigcup E_{i} \supset A, \operatorname{diam} E_{i} \leq \delta\right\} .
$$

A set $A \subset \mathbb{R}$ is an $h$-set if $0<\mathcal{H}^{h}(A)<+\infty$.

Given $0 \leq s \leq 1$, it is not difficult to construct a Cantor set that is an $s$-set. It is also known that not every Cantor set of dimension $s$ is an $s$-set. So should a set of dimension $s$ but Hausdorff measure zero or infinity be considered $s$-dimensional?

Hausdorff proposed the $h$-measure that bears his name to further the investigation of non-s-sets. In this paper we prove that every Cantor set $C$ on the line associated to a sequence of nonincreasing positive real numbers is an $h$-set for some continuous concave function $h$. We explicitly construct $h$ in terms of the sequence that defines the Cantor set. In other words, for every sequence, the set with the largest Hausdorff dimension is also an $h$-set for some appropriate function.

The study of Cantor sets through the decay of the complementary intervals was initiated by Borel in 1948 [Bor49] and continued by Besicovitch and Taylor in their seminal paper [BT54]. The present paper extends some of their results.

Tricot [Tri81] and Falconer [Fal97] obtained results associating properties of the gaps of a Cantor set with its box dimension. (See also [Tri95]). In [CMPS03] the particular case of the sequence $x^{p}$ was thoroughly analyzed.

Throughout the paper, we will use the notation $\operatorname{dim} A$ for the Hausdorff dimension of a set $A$, since it is the only concept of dimension that we are considering. The Hausdorff $s$-measure of a set $A$ will be denoted by $\mathcal{H}^{s}(A)$.

\section{Cantor sets associated to a sequence}

We will now assign to each summable sequence of positive numbers a unique Cantor set with gaps whose lengths correspond to the terms of this sequence. Let $a=\left\{a_{k}\right\}$, for $k=1,2, \ldots$, be a sequence of positive real numbers such that $\sum a_{k}=S_{a}<\infty$. Let $I$ be an interval of length $|I|=S_{a}$. We first remove from $I$ an open interval of length $a_{1}$, whose position will be clear in a moment. We next remove from the left remaining interval an interval of length $a_{2}$ and from the right an interval of length $a_{3}$. We continue in this way, removing at the $i$-th step $2^{i-1}$ intervals from left to right. It is 
easy to see that we end up with a Cantor set, which we will call $C_{a}$. Since $\sum a_{k}=|I|$, there is only one choice for the location of each interval to be removed in the construction, and $C_{a}$ is well-defined.

The gap of $C_{a}$ associated with the term $a_{k}$ will be denoted $g_{a_{k}}$. If $g$ and $g^{\prime}$ are gaps, we will say that $g<g^{\prime}$ if all $x \in g, y \in g^{\prime}$ satisfy $x<y$. Given a sequence $a$ and its associated Cantor set $C_{a}$, we define a $c u t$ of $C_{a}$ to be a partition of $\mathbb{N}=L \cup R$ such that

$$
g_{a_{\ell}}<g_{a_{r}} \text { for all } \ell \in L, r \in R .
$$

We will allow $L$ or $R$ to be empty. The following lemma is an immediate consequence of the definitions.

Lemma 1. Every point in $C_{a}$ defines a cut and, conversely, every cut of $C_{a}$ defines a unique point of $C_{a}$.

Let $C_{a}$ and $C_{b}$ be Cantor sets associated to sequences $a$ and $b$ respectively. As a result of the definition of $C_{a}$ and $C_{b}$ it is clear that, for any $n, m \in \mathbb{N}$,

$$
g_{a_{n}}<g_{a_{m}} \text { implies } g_{b_{n}}<g_{b_{m}} \text {. }
$$

This implies that if $(L, R)$ defines a cut of $C_{a}$, it also defines a cut of $C_{b}$.

If $x \in C_{a}$ is defined by a cut $(L, R)$, then $x=\sum_{n \in L}\left|g_{a_{n}}\right|$.

\section{Equivalences of Cantor sets}

The previous considerations allow us to define a natural map $\pi_{a b}$ from $C_{a}$ into $C_{b}$, assigning to the point $x \in C_{a}$ the point $y \in C_{b}$ defined by the same cut associated to $x$, i.e., if $L_{a}(x)=\left\{n \in \mathbb{N}: g_{a_{n}} \subset[0, x]\right\}$, then

$$
y=\pi_{a b}(x)=\sum_{n \in L_{a}(x)}\left|g_{b_{n}}\right| \text {. }
$$

Observe that $y$ can be written also as

$$
y=\sum_{n \in L_{b}(y)}\left|g_{b_{n}}\right|, \quad \text { with } L_{b}(y)=\left\{n \in \mathbb{N}: g_{b_{n}} \subset[0, y]\right\} .
$$

The map $\pi_{a b}: C_{a} \rightarrow C_{b}$ is one-to-one and onto. It can be extended linearly to a one-to-one map from $\left[0, S_{a}\right]$ into $\left[0, S_{b}\right]$, by mapping the gap $g_{a_{n}}$ linearly into the gap $g_{b_{n}}$.

Note that $\pi$ is an increasing function, since given $x, y \in C_{a}$ with $x<y$, we have

$$
\pi_{a b}(y)-\pi_{a b}(x)=\sum_{n \in L_{a}(y)} b_{n}-\sum_{n \in L_{a}(x)} b_{n}=\sum_{n \in L_{a}(y) \backslash L_{a}(x)} b_{n}=\sum_{\left\{n: g_{a_{n}} \subset[x, y]\right\}} b_{n}>0 .
$$

Thus $\pi_{a b}$ is increasing on $C_{a}$. This implies that $\pi_{a b}$ is increasing on $\left[0, S_{a}\right]$. Since $\pi_{a b}:\left[0, S_{a}\right] \rightarrow\left[0, S_{b}\right]$ is onto, it must be continuous and consequently $\pi_{a b}^{-1}:\left[0, S_{b}\right] \rightarrow\left[0, S_{a}\right]$ is also continuous.

We have therefore proved the following proposition: 
Proposition 1. If $C_{a}$ and $C_{b}$ are the Cantor sets associated to arbitrary sequences $a$ and $b$, then the map $\pi_{a b}:\left[0, S_{a}\right] \rightarrow\left[0, S_{b}\right]$ is increasing, one to one, onto and bicontinuous. Furthermore $\pi_{a b}\left(C_{a}\right)=C_{b}$.

Definition 1. We define an order relation $\prec$ between summable sequences of positive terms as follows: if $a$ and $b$ are two such sequences, we set

$$
a \prec b \quad \text { if there exists } k>0 \text { such that } \frac{a_{n}}{b_{n}}<k \text { for all } n \in \mathbb{N} \text {. }
$$

In this case we say that $a$ is of lower order than $b$. If $a \prec b$ and $b \prec a$ we say that $a$ and $b$ are of the same order and we write $a \sim b$. Note that

$$
a \sim b \Longleftrightarrow k_{1}<\frac{a_{n}}{b_{n}}<k_{2} \text { for all } n \in \mathbb{N}
$$

for some constants $k_{1}, k_{2}>0$.

We will need the following result from [CMPS03]:

Proposition 2. Let $a=\left\{a_{k}\right\}_{k \in \mathbb{N}}$ be defined by $a_{k}=\left(\frac{1}{k}\right)^{p}$, with $p>1$. Then $\operatorname{dim}\left(C_{a}\right)=1 / p$, and moreover, $C_{a}$ is a $(1 / p)$-set; precisely,

$$
\frac{1}{8}\left(\frac{2^{p}}{2^{p}-2}\right)^{1 / p} \leq \mathcal{H}^{1 / p}\left(C_{a}\right) \leq\left(\frac{1}{p-1}\right)^{1 / p} .
$$

The following notation is convenient in the proofs below.

Notation 1. We write $\lambda^{(p)}$ for the sequence whose $n$-th term is $n^{-p}$.

Theorem 1. Let $C_{a}$ and $C_{b}$ be Cantor sets associated to the sequences a and $b$.

(1) If $a \prec b$ then $\operatorname{dim}\left(C_{a}\right) \leq \operatorname{dim}\left(C_{b}\right)$; thus $\operatorname{dim}\left(C_{a}\right)=\operatorname{dim}\left(C_{b}\right)$ if $a \sim b$.

(2) There exist sequences $a=\left\{a_{n}\right\}$ and $b=\left\{b_{n}\right\}$ such that

$$
\liminf \frac{a_{n}}{b_{n}}=0 \quad \text { and } \quad \operatorname{dim}\left(C_{a}\right)=\operatorname{dim}\left(C_{b}\right) .
$$

Proof of theorem. For part (1), if $a \prec b$, we will show that the map $\pi_{b a}$ defined above is Lipschitz. Given $x, y \in C_{b}$ with $x<y$, we have

$$
\pi_{b a}(y)-\pi_{b a}(x)=\sum_{\left\{n: g_{b_{n}} \subset[x, y]\right\}} a_{n} \leq k \sum_{\left\{n: g_{b_{n}} \subset[x, y]\right\}} b_{n}=k(y-x) .
$$

Then $\operatorname{dim}\left(C_{a}\right)=\operatorname{dim}\left(\pi_{b a}\left(C_{b}\right)\right)$. By an elementary property of Hausdorff dimension we obtain $\operatorname{dim}\left(\pi_{a b}\left(C_{b}\right)\right) \leq \operatorname{dim}\left(C_{b}\right)$, proving (1).

For part (2), consider a sequence $a=\left\{a_{n}\right\}$ such that for some fixed $p>1$

$$
\lim _{n \rightarrow \infty} \frac{a_{n}}{n^{-p}}=0 \quad \text { and } \quad \lim _{n \rightarrow \infty} \frac{n^{-q}}{a_{n}}=0, \quad \text { for all } q>p .
$$

The maps $\pi_{\lambda^{(p)} a}: C_{\lambda^{(p)}} \rightarrow C_{a}$ and $\pi_{a \lambda^{(q)}}: C_{a} \rightarrow C_{\lambda^{(q)}}$ are Lipschitz, as can be seen by means of an argument similar to that of part (1). This implies that $q-1 \leq \operatorname{dim}\left(C_{a}\right) \leq p-1$ for all $q>p$. Then $\operatorname{dim}\left(C_{a}\right)=1 / p$. 


\section{Computation of Hausdorff dimensions}

In this section we define some indices associated with a summable sequence. These numbers can be considered as a measure of the decay rate of the sequence. We then compare their values with the dimension of the associated Cantor set.

For a sequence $a=\left\{a_{n}\right\}$, define

$$
\begin{aligned}
& \beta(a)=\inf \left\{s: 0<s, a \prec \lambda^{(1 / s)}\right\}, \\
& \gamma(a)=\sup \left\{s: 0<s, \lambda^{(1 / s)} \prec a\right\}, \\
& \delta(a)=\inf \left\{s: 0<s \leq 1, \sum_{n} a_{n}^{s}<\infty\right\} .
\end{aligned}
$$

Of these constants, only $\delta$ is invariant under rearrangements; $\beta$ and $\gamma$ are not. Since we know that for the sequence $\lambda^{(p)}$ rearrangements can indeed change the dimension (see [CMPS03]), we have to discard the intuition that $\delta(a)=\operatorname{dim}\left(C_{a}\right)$.

A historical survey of various indices associated with the decay of gaps (when $a_{n}$ decreases) and the box dimension is given by Tricot in [Tri81], together with more complete results. In particular he shows that

$$
\gamma(a)=\underline{\lim } \frac{-\log n}{\log a_{n}} \quad \text { and } \quad \beta(a)=\varlimsup \frac{-\log n}{\log a_{n}},
$$

and if $a=\left\{a_{n}\right\}$ is monotonic decreasing, then $\delta(a)=\beta(a)$.

Proposition 3. Let a be a summable sequence of positive terms.

(1) $\gamma(a) \leq \operatorname{dim}\left(C_{a}\right) \leq \beta(a)$.

(2) $\gamma(a) \leq \delta(a) \leq \beta(a)$.

Proof. Part (1) is a consequence of Theorem 1 and the definition of $\gamma(a)$ and $\beta(a)$.

For part (2), choose $s>0$ such that $a \prec \lambda^{(1 / s)}$, which is to say

$$
a_{n} \leq \frac{c}{n^{1 / s}} \text { for some } c>0 \text { and every } n \text {. }
$$

Then, for some other constant $c^{\prime}$,

$$
a_{n}^{s+\epsilon} \leq \frac{c^{\prime}}{n^{(s+\epsilon) / s}} \quad \text { for every } n
$$

which implies that $s+\epsilon \geq \delta(a)$ for all $\epsilon>0$; thus $\delta(a) \leq \beta(a)$. Furthermore, for each $\epsilon \geq 0$ we have

$$
c n^{-\frac{1}{\gamma(a)-\epsilon}} \leq a_{n} \quad \text { for some } c \text { and every } n,
$$

and so

$$
\sum_{n} a_{n}^{\gamma(a)-\epsilon}=+\infty
$$


which implies that $\delta(a) \geq \gamma(a)-\epsilon$. Since $\epsilon$ is arbitrary, we conclude that $\delta(a) \geq \gamma(a)$.

A consequence of the proposition and Tricot's result is that if $a$ is a monotone nonincreasing summable sequence of positive terms and $\widetilde{a}$ is any rearrangement of $a$ then $\beta(a)=\delta(a)=\delta(\widetilde{a}) \leq \beta(\widetilde{a})$.

Another immediate consequence of the definition of $\gamma(a)$ and $\beta(a)$ is:

Property. Let $a$ be a summable sequence of positive terms. If $0<b<\beta(a)$ then $\varlimsup_{n \rightarrow \infty} n^{1 / b} a_{n}=+\infty$, and if $\gamma(a) \leq b$ then $\underline{\lim }_{n \rightarrow \infty} n^{1 / b} a_{n}=0$.

This tells us that if we take a rearrangement $\widetilde{a}$ of a monotone nonincreasing sequence $a$ such that $\beta(a) \neq \beta(\widetilde{a})$ (so that $\beta(a)<\beta(\widetilde{a})$ ), then $\varlimsup_{n \rightarrow \infty} n^{1 / \beta(a)} \widetilde{a}_{n}=+\infty$. Therefore, if $\widetilde{a}$ is a rearrangement of a monotonic nonincreasing sequence $a$, then $\operatorname{dim}\left(C_{\widetilde{a}}\right) \leq \beta(a)<\beta(\widetilde{a})$.

4.1. Monotone nonincreasing sequences. For a nonincreasing sequence $a$, we already know that $\delta(a)=\beta(a)$. In addition, by Proposition 3, we know that $\gamma(a) \leq \operatorname{dim}\left(C_{a}\right) \leq \beta(a)$. Therefore, if $\lim \left(\log a_{n} / \log n\right)=\ell$, we have $\operatorname{dim}\left(C_{a}\right)=-1 / \ell$.

This result extends the result of Falconer [Fal97, p. 55]. Moreover, that author shows that if that limit does not exist then the upper and lower box-dimensions disagree.

In this case, however, we still want to determine the dimension of $C_{a}$. To this end, we introduce two new constants associated to the sequence $a$. Set $r_{n}=\sum_{j \geq n} a_{j}$. Using an argument analogous to the one used in [CMPS03], one can see that the $s$-Hausdorff measure of $C_{a}$ is bounded by

$$
H^{s}\left(C_{a}\right) \leq c \underline{\lim } n\left(\frac{r_{n}}{n}\right)^{s} .
$$

We therefore define two constants associated to the sequence $a$ :

$$
\begin{aligned}
& \tau(a)=\inf \left\{s>0: \underline{\lim } n\left(\frac{r_{n}}{n}\right)^{s}<+\infty\right\}, \\
& \alpha(a)=\underline{\lim } \alpha_{n}, \quad \text { where } n\left(\frac{r_{n}}{n}\right)^{\alpha_{n}}=1 .
\end{aligned}
$$

Note. The constant $\alpha$ associated to a monotone sequence $a$ was introduced in [BT54]. The authors show that $\operatorname{dim}\left(C_{\widetilde{a}}\right) \leq \alpha(a)$, where $\widetilde{a}$ is any rearrangement of $a$.

It is interesting to remark that $\varlimsup$ im $\alpha_{n}$ was introduced already in 1948 by Emil Borel with the name of logarithmic density.

From results in the seminal paper by Besicovitch and Taylor [BT54], one can conclude that $\operatorname{dim}\left(C_{a}\right)=\alpha(a)$ for a monotonic nonincreasing sequence (see [CHM03]), and that for each $t$ and $\beta$ with $0 \leq t \leq \beta$ there is a monotone nonincreasing sequence $a=\left\{a_{n}\right\}$ such that $\beta(a)=\beta$ and $\alpha(a)=$ $t$. Our next proposition, however, expresses the surprising result that if $\gamma(a)$ is strictly smaller than $\beta(a)$, then $\alpha(a)$ has a smaller than expected bound. 
Proposition 4. With notation as above, for a nonincreasing sequence a,

$$
\alpha(a)=\tau(a) \quad \text { and } \quad \alpha(a) \leq \frac{\gamma(a)}{1-\beta(a)+\gamma(a)} .
$$

Proof. We first show that $\alpha(a) \leq \tau(a)$. Let $s>0$ be such that $\underline{\lim } n\left(\frac{r_{n}}{n}\right)^{s}<$ $+\infty$. Then

$$
n\left(\frac{r_{n}}{n}\right)^{s}=n\left(\frac{r_{n}}{n}\right)^{\alpha_{n}}\left(\frac{r_{n}}{n}\right)^{s-\alpha_{n}}=\left(\frac{r_{n}}{n}\right)^{s-\alpha_{n}} .
$$

So $\underline{\lim }\left(\frac{r_{n}}{n}\right)^{s-\alpha_{n}}<+\infty$. Since $\lim \left(\frac{r_{n}}{n}\right)^{-1 / k}=+\infty$ for each fixed $k>0$, there must exist a subsequence $\alpha_{n_{k}}$ such that $\alpha_{n_{k}}<s+1 / k$ for all $k$. We have $\alpha(a)=\underline{\lim }_{n} \alpha_{n} \leq \underline{\lim } \alpha_{n_{k}} \leq s$, and therefore $\alpha(a) \leq \tau(a)$.

For the converse, $\tau(a) \leq \alpha(a)$, assume that $\alpha(a)<\tau(a)$, and consider $s$ such that $\alpha(a)<s<\tau(a)$. Let $\left\{a_{n_{k}}\right\}$ be such that $\lim _{k} a_{n_{k}}=\alpha(a)$ and $a_{n_{k}}<s$ for all $k$. Then

$$
+\infty=\underline{\lim }_{n} n\left(\frac{r_{n}}{n}\right)^{s}=\underline{\lim }_{k} n_{k}\left(\frac{r_{n_{k}}}{n_{k}}\right)^{s}=\underline{\lim }_{k}\left(\frac{r_{n_{k}}}{n_{k}}\right)^{s-\alpha_{n_{k}}}=0
$$

(since $s-\alpha_{n_{k}}>c>0$ for some $c$ and for all $k$ ). This contradiction shows that $\alpha(a)=\tau(a)$.

For the other inequality, note that if $\gamma(a)=\beta(a)$, then

$$
\frac{\gamma(a)}{1-\beta(a)+\gamma(a)}=\gamma(a)
$$

and there is nothing to prove. However, if $\gamma(a)<\beta(a)$, then

$$
\frac{\gamma(a)}{1-(\beta(a)-\gamma(a))}<\beta(a)
$$

To show that $\alpha(a)$ satisfies the desired inequality, we prove

$$
\alpha(a) \leq \frac{\gamma(a)+\varepsilon}{1-(\beta(a)-\gamma(a)-\varepsilon)} \quad \text { for each } \varepsilon>0
$$

To this end, we will show that for each $\varepsilon>0$, there is a subsequence $\left\{a_{n_{k}}\right\}_{k}$ of $\left\{a_{n}\right\}_{n}$ for which $r_{n_{k}}$ is at most $O\left(n_{k}^{-\frac{1-\beta(a)}{(\gamma(a)+\varepsilon)}}\right)$.

Fix $\beta(a)-\gamma(a) \geq \varepsilon>0$, and set $\gamma_{\varepsilon}=\gamma(a)+\varepsilon$. We see immediately from the definition of $\gamma(\bar{a})$ that there is a subsequence $n_{k}$ such that $a_{n_{k}} \leq n_{k}^{-1 / \gamma_{\varepsilon}}$. This is the subsequence that we desire.

Since $a_{n}$ is monotone, we can estimate $r_{n_{k}}$ from above. Fix $n_{k}$. Define a new sequence $\left\{b_{n}\right\}_{n}$ in the following way:

$$
b_{j}= \begin{cases}a_{j} & \text { for } j \leq n_{k}, \\ n_{k}^{-1 / \gamma_{\varepsilon}} & \text { for } n_{k} \leq j<\left\lceil n_{k}^{\beta(a) / \gamma_{\varepsilon}}\right\rceil, \\ j^{-1 / \beta(a)} & \text { for all larger } j .\end{cases}
$$


Here as usual $\lceil x\rceil$ stands for the smallest integer that is larger or equal than $x$. So we have that $a_{j} \leq b_{j}$ for all $j$, and therefore $\sum_{j \geq n_{k}} a_{j} \leq \sum_{j \geq n_{k}} b_{j}$.

We can estimate that

$$
\sum_{j=n_{k}}^{\left\lceil n_{k}^{\beta / \gamma_{\varepsilon}}\right\rceil} b_{j}=\frac{\left\lceil n_{k}^{\beta(a) / \gamma_{\varepsilon}}\right\rceil-n_{k}}{n_{k}^{1 / \gamma_{\varepsilon}}} \sim n_{k}^{(\beta(a)-1) / \gamma_{\varepsilon}} \text { for } k \text { large enough, }
$$

and, using an integral comparison, we see that

$$
\sum_{j \geq\left\lceil n_{k}^{\left.\beta(a) / \gamma_{\varepsilon}\right\rceil}\right.} b_{j}=C\left(n_{k}^{\beta(a) / \gamma_{\varepsilon}}\right)^{(\beta(a)-1) / \beta(a)} .
$$

Since both of these terms are $O\left(n_{k}^{(\beta(a)-1) / \gamma_{\varepsilon}}\right)$, we have

$$
\alpha(a) \leq \frac{\gamma(a)+\varepsilon}{1-(\beta(a)-\gamma(a)-\varepsilon)} \quad \text { for every } \varepsilon .
$$

In $[$ Tri95] it is proved that

$$
\beta(a)=\varlimsup-\frac{\log n}{\log a_{n}}=\varlimsup \alpha_{n} .
$$

Proposition 4 shows this is false, in general, for the $\underline{\text { lim }}$. Moreover, we know that there are no sequences $a$, with $\gamma(a)<\beta(a)$ and

$$
\frac{\gamma(a)}{1-\beta(a)+\gamma(a)}<\operatorname{dim}\left(C_{a}\right) \leq \beta(a)
$$

So the question now is whether there exists a sequence $a$ such that

$$
\gamma(a) \leq \operatorname{dim}\left(C_{a}\right) \leq \frac{\gamma(a)}{1-\beta(a)+\gamma(a)} .
$$

The next proposition answers this question completely and emphasizes the asymmetry between the $\varlimsup$ lim and the $\underline{\underline{\lim }}$.

Proposition 5. Let $0<\gamma \leq \beta \leq 1$ be given. For any number $t$ such that $\gamma \leq t \leq \gamma /(1-\beta+\gamma)$, there is a monotonic nonincreasing sequence a such that $\operatorname{dim}\left(C_{a}\right)=t$ and

$$
\gamma(a)=\gamma \quad \text { and } \quad \beta(a)=\beta .
$$

Proof. Let $0 \leq s \leq 1$, and define

$$
f(s)=\frac{\gamma(1-s \beta)}{1-\beta+\gamma(1-s)} .
$$

For each $s$ we construct a monotonic nonincreasing sequence $a^{(s)}$ satisfying $\operatorname{dim}\left(C_{a^{(s)}}\right)=f(s), \gamma\left(a^{(s)}\right)=\gamma$, and $\beta\left(a^{(s)}\right)=\beta$. Since $f$ is decreasing, $f(0)=\frac{\gamma}{1-\beta+\gamma}$ and $f(1)=\gamma$, there exists for any $t \in\left[\gamma, \frac{\gamma}{1-\beta+\gamma}\right]$ an $s_{t}$ so that $\operatorname{dim}\left(C_{a\left(s_{t}\right)}\right)=t$. 
To construct such a sequence, let

$$
R=\frac{1-\gamma s}{1-\beta s} \frac{\beta}{\gamma}
$$

and define $p_{n}=2^{R^{n}}$, for $n=0,1,2, \ldots$ Define the sequence $a^{(s)}=\left\{a_{n}\right\}$ as follows: $a_{0}=a_{1}=1$ and

$$
a_{j}=\left(p_{n}\right)^{-(1-s \gamma) / \gamma} j^{-s} \quad \text { when } p_{n} \leq j<p_{n+1} \text {. }
$$

Notice that $a_{p_{n}}=p_{n}^{-1 / \gamma}$ and

$$
a_{\left(p_{n+1}-1\right)}=p_{n}^{-(1-s \gamma) / \gamma}\left(p_{n}^{R}-1\right)^{-s} \sim p_{n+1}^{-1 / \beta} .
$$

Furthermore, $n^{-1 / \gamma} \leq a_{n} \leq n^{-1 / \beta}$. Hence $\gamma\left(a^{(s)}\right)=\gamma$ and $\beta\left(a^{(s)}\right)=\beta$, so $a$ satisfies the desired conditions. In addition $a^{(s)}$ satisfies

$$
\alpha\left(a^{(s)}\right)=\frac{\gamma(1-s)}{(1-\beta)+\gamma(1-s)}=f(s) .
$$

To show this, we estimate $r_{p_{n}}$. We see that

$$
r_{p_{n}}=\sum_{p_{n} \leq j<p_{n+1}} a_{j}+\sum_{j \geq p_{n+1}} a_{j} \sim C p_{n}^{-\frac{1-s \gamma}{1-s \beta} \frac{1-\beta}{\gamma}}
$$

so that

$$
\alpha\left(a^{(s)}\right) \leq \frac{\gamma(1-s \beta)}{(1-\beta)+\gamma(1-s)} .
$$

To see the other inequality observe that for $i \in \mathbb{N}$ with $p_{n}<i<p_{n+1}$, we have

$$
\alpha_{i}=\frac{\ln (1 / i)}{\ln \left(r_{i} / i\right)} \geq \frac{\ln \left(1 / p_{n}\right)}{\ln \left(r_{p_{n}} / p_{n}\right)=\alpha_{p_{n}}} .
$$

This estimate is obtained by noting that if $\tau$ is such that $i=p_{n}^{\tau}(1<\tau<R)$, then

$$
r_{i} \approx p_{n}^{-\frac{1-s \gamma}{\gamma}}\left(p_{n}^{R(1-s)}-p_{n}^{\tau(1-s)}\right)+p_{n}^{R^{2}(1-s)-\frac{R}{\gamma}},
$$

and since $1<\tau<R$, by (2) asymptotically we have that $r_{i} / r_{p_{n}} \rightarrow 1$. Thus, for large enough values, we know that $1<\ln r_{i} / \ln r_{p_{n}}<\tau$, which is equivalent to the desired inequality. Therefore $\operatorname{dim}\left(C_{a^{(s)}}\right)=f(s)$.

We summarize in the next theorem the main results of this section.

Theorem 2. Let $a=\left\{a_{n}>0\right\}$ be a summable sequence.

(1) $0 \leq \gamma(a) \leq \operatorname{dim}\left(C_{a}\right) \leq \alpha(a) \leq \frac{\gamma(a)}{1-\beta(a)+\gamma(a)} \leq \beta(a)$. In particular, when the sequence $a$ is nonincreasing we have $\operatorname{dim}\left(C_{a}\right)=\alpha(a)$. 
(2) Given numbers $\alpha, \beta$ and $\gamma$ with

$$
0 \leq \gamma \leq \alpha \leq \frac{\gamma}{1-\beta+\gamma} \leq \beta \leq 1,
$$

there exists a summable sequence a (which can be chosen to be nonincreasing) such that $\gamma(a)=\gamma, \alpha(a)=\alpha$ and $\beta(a)=\beta$.

Given a nonincreasing sequence $a$ it could happen that the $\alpha(a)$-Hausdorff measure of the associated Cantor set $C_{a}$ is zero or infinite. In the next section we will see that we can still say something in this case.

\section{Dimension function}

To analyze this situation it will be useful to refine the notion of dimension, in the spirit of Hausdorff's original work. Throughout this section we fix a monotonic nonincreasing sequence $a=\left\{a_{k}\right\}$ of positive terms such that $\sum a_{k}=1$.

We associate to $a$ another nonincreasing sequence:

$$
b=\left\{b_{n}\right\} \quad \text { with } \quad b_{n}=\frac{r_{n}}{n}, \quad \text { where } r_{n}=\sum_{j=n}^{\infty} a_{j} \text { as before. }
$$

Fix a decreasing function $f:[1,+\infty) \rightarrow \mathbb{R}$ such that $f(k)=b_{k}$, for example

$$
f(x)=b_{k}(k+1-x)+b_{k+1}(x-k), \quad x \in[k, k+1) .
$$

Then define

$$
h(t)= \begin{cases}1 / f^{-1}(t), & t \in\left(0, b_{1}\right], \\ 0, & t=0 .\end{cases}
$$

Then $h$ is a nondecreasing, concave function and $h\left(b_{k}\right)=1 / k$. This function will be useful for determining the dimension of the Cantor set $C_{a}$.

We will need some auxiliary results and (more!) notation.

Let $W$ denote the set of binary words of finite length:

$$
W=\{e\} \cup\left\{w_{1} \cdots w_{r}: w_{i} \in\{0,1\}, r \in \mathbf{N}\right\},
$$

where $e$ denotes the empty word. If $w, w^{\prime} \in W$ let $w w^{\prime}$ be the concatenation of $w$ and $w^{\prime}$, and $|w|$ the length $w$, with $|e|=0$. Let $W^{*}$ denote the set of words of positive length. Given $w$, either an infinite binary word or a finite binary word of length at least $k$, we will denote by $w(k)$ the truncation $w_{1} \cdots w_{k}$.

It is convenient to use the elements of $W$ to describe the intervals of our Cantor set $C_{a}$. Let $I_{e}$ denote the initial interval. $\left(I_{e}=I_{0}^{0}\right)$. If $w \in W$, $|w|=k$ and $I_{w}$ is an interval of step $k$ in the construction, denote by $I_{w 0}$ and $I_{w 1}$ the left and right intervals obtained by removing the open interval from $I_{w}$. 
In this way, if $I_{w}$ is an interval of step $|w|$, with

$$
I_{w}=I_{\sum_{j=1}^{|w|} w_{j} 2^{k-j}}^{|w|},
$$

and if $w^{\prime} \in W$, we see that $I_{w w^{\prime}}$ is an interval of step $\left|w w^{\prime}\right|$, which we say is related to $I_{w}$.

It is worthwhile to note at this stage that in the case of a monotonic nonincreasing sequence, the lengths of $I_{w}$ also form a nonincreasing sequence.

For the sequence $b_{n}$ defined on the previous page we will now denote by $b_{w}$ the element of the sequence corresponding to $b_{\ell}$, with $\ell=2^{k}+\sum_{j=1}^{k} w_{j} 2^{k-j}$ and $k=|w|$.

In particular,

$$
\text { if } \quad b_{w}=b_{2^{k}+l} \text { then } \quad b_{w w^{\prime}}=b_{2^{k^{\prime}}\left(2^{k}+l\right)+s},
$$

where $l=\sum_{j=1}^{k} w_{j} 2^{k-j}$ with $k=|w|$ and $s=\sum_{j=1}^{k^{\prime}} w_{j}^{\prime} 2^{k^{\prime}-j}$ with $k^{\prime}=\left|w^{\prime}\right|$.

Lemma 2. With the above notation, for every $k \geq 1$, and $w, \widetilde{w}$ of length $k$, and any $w^{\prime}$,

$$
\frac{1}{2} \frac{h\left(b_{w w^{\prime}}\right)}{h\left(b_{w}\right)} \leq \frac{h\left(b_{\widetilde{w} w^{\prime}}\right)}{h\left(b_{\widetilde{w}}\right)} \leq 2 \frac{h\left(b_{w w^{\prime}}\right)}{h\left(b_{w}\right)} .
$$

In particular, for any $w^{\prime}$ we have $h\left(b_{w w^{\prime}}\right) \leq 4 h\left(b_{w}\right)$.

Proof. Recall that $h\left(b_{\ell}\right)=1 / \ell$ and let $k^{\prime}=\left|w^{\prime}\right|$. Define

$$
l=\sum_{j=0}^{k} w_{j} 2^{k-j}, \quad r=\sum_{j=0}^{k} \widetilde{w_{j}} 2^{k-j} \quad \text { and } \quad s=\sum_{j=0}^{k^{\prime}} w_{j}^{\prime} 2^{k^{\prime}-j} .
$$

Then, by (3),

$$
\frac{h\left(b_{w w^{\prime}}\right)}{h\left(b_{w}\right)}=\frac{2^{k}+l}{2^{k^{\prime}}\left(2^{k}+l\right)+s} \quad \text { and } \quad \frac{h\left(b_{\widetilde{w} w^{\prime}}\right)}{h\left(b_{\widetilde{w}}\right)}=\frac{2^{k}+r}{2^{k^{\prime}}\left(2^{k}+r\right)+s} .
$$

Now noting that

$$
\frac{1}{2} \leq \frac{2^{k}+r}{2^{k}+l} \leq 2
$$

we obtain the desired result.

For the second inequality just note that $h$ is nondecreasing and therefore the right-hand side is less or equal than 2 for any $w^{\prime}$.

These bounds of the ratios of $h\left(b_{k}\right)$ will be useful for defining a measure on $C_{a}$. Since the construction of this Cantor set relies on the size of the gaps, it will be useful to define a measure depending on the size of the gaps.

Proposition 6. There exists a probability measure $\mu_{h}$ supported on $C_{a}$ such that, for every $k \geq 1$ and $0 \leq \ell \leq 2^{k}-1$,

$$
\frac{1}{4} h\left(b_{2^{k}+\ell}\right) \leq \mu_{h}\left(I_{\ell}^{k}\right) \leq 2 h\left(b_{2^{k}+\ell}\right) .
$$


Proof. For $m \geq 1$ consider the probability measure $\mu_{m}$ supported on the intervals $I_{\ell}^{m}$ of level $m$ and such that

$$
\mu_{m}\left(I_{t}^{m}\right)=\frac{h\left(b_{2^{m}+t}\right)}{\sum_{j=0}^{2^{m}-1} h\left(b_{2^{m}+j}\right)} .
$$

If $k \leq m$ and $w=w_{1} \ldots w_{k}$ is such that $\sum_{j=0}^{k} w_{j} 2^{k-j}=t$, we have

$$
\mu_{m}\left(I_{t}^{k}\right)=\mu_{m}\left(I_{w}\right)=\sum_{\left|w^{\prime}\right|=m-k} \mu_{m}\left(I_{w w^{\prime}}\right)
$$

and hence

$$
\left(\sum_{j=0}^{2^{m}-1} h\left(b_{2^{m}+j}\right)\right) \mu_{m}\left(I_{t}^{k}\right)=\sum_{\left|w^{\prime}\right|=m-k} h\left(b_{w w^{\prime}}\right) .
$$

But by the bounds found in Lemma 2,

$$
h\left(b_{w w^{\prime}}\right) \leq 2 h\left(b_{w}\right) \frac{h\left(b_{\widetilde{w} w^{\prime}}\right)}{h\left(b_{\widetilde{w}}\right)}, \text { for all } \widetilde{w} \text { such that }|\widetilde{w}|=|w|=k .
$$

Hence, recalling the definition of $w$, we obtain (from (5)), that for all $\widetilde{w}$ such that $|\widetilde{w}|=k$,

$$
\left(h\left(b_{\widetilde{w}}\right) \sum_{j=0}^{2^{m}-1} h\left(b_{2^{m}+j}\right)\right) \mu_{m}\left(I_{t}^{k}\right) \leq 2 h\left(b_{w}\right) \sum_{\left|w^{\prime}\right|=m-k} h\left(b_{\widetilde{w} w^{\prime}}\right),
$$

and therefore

$$
\begin{aligned}
\left(\sum_{|\widetilde{w}|=k} h\left(b_{\widetilde{w}}\right) \sum_{j=0}^{2^{m}-1} h\left(b_{2^{m}+j}\right)\right) \mu_{m}\left(I_{t}^{k}\right) & \leq 2 h\left(b_{w}\right)\left(\sum_{|\widetilde{w}|=k} \sum_{\left|w^{\prime}\right|=m-k} h\left(b_{\widetilde{w} w^{\prime}}\right)\right) \\
& =2 h\left(b_{w}\right) \sum_{j=0}^{2^{m}-1} h\left(b_{2^{m}+j}\right),
\end{aligned}
$$

which yields

$$
\mu_{m}\left(I_{\ell}^{k}\right) \leq 2 \frac{h\left(b_{2^{k}+\ell}\right)}{\sum_{j=0}^{2^{k}-1} h\left(b_{2^{k}+j}\right)}, \quad k \leq m
$$

But noting that

$$
\frac{1}{2} \leq \sum_{j=0}^{2^{m}-1} h\left(b_{2^{m}+j}\right) \leq 1
$$

and using the other inequality of Lemma 2, we finally obtain $\frac{1}{2} h\left(b_{2^{k}+\ell}\right) \leq \mu_{m}\left(I_{\ell}^{k}\right) \leq 4 h\left(b_{2^{k}+\ell}\right) \quad$ for every $1 \leq k \leq m, 0 \leq \ell \leq 2^{k}-1$. 
Now let $\mu_{h}$ be the weak*-limit of $\mu_{m}$. then (see for example [Mat95]) for every $1 \leq k, 0 \leq \ell \leq 2^{k}-1$,

$$
\frac{1}{2} h\left(b_{2^{k}+\ell}\right) \leq \mu_{h}\left(I_{\ell}^{k}\right) \leq 4 h\left(b_{2^{k}+\ell}\right) .
$$

We are now ready to prove our main result. Recall that an $h$-set was defined in Equation (1) of the introduction.

Theorem 3. Let $a=\left\{a_{k}\right\}$ be a nonincreasing sequence of positive terms such that $\sum a_{k}=1$ and $C_{a}$ the associated Cantor set. Then $C_{a}$ is an h-set. Moreover

$$
\frac{1}{32} \leq \mathcal{H}^{h}\left(C_{a}\right) \leq 1,
$$

where $\mathcal{H}^{h}$ is the Hausdorff measure associated to $h$, and $h$ is the dimension function defined on page 54 .

Proof. For the upper bound, fix $\delta>0$ and let $n_{0}$ be such that $n \geq n_{0}$, $r_{n}=\sum_{j \geq n} a_{j}<\delta$. Then the intervals $E_{1}, \ldots, E_{n}$ remaining after the gaps associated to $a_{1}, \ldots, a_{n-1}$ are removed form a $\delta$-covering of $C_{a}$, and since $h$ is concave, we have

$$
\sum_{i=1}^{n} h\left(\left|E_{i}\right|\right) \leq n h\left(\frac{\left|E_{1}\right|+\cdots+\left|E_{n}\right|}{n}\right)=n h\left(\frac{r_{n}}{n}\right)=1 .
$$

Therefore $\mathcal{H}^{h}\left(C_{a}\right) \leq 1$.

For the lower bound, the idea is to try to use the measure $\mu_{h}$ and apply a generalized version of the mass transfer principle. To this end, let $U$ be any open set with $\operatorname{diam}(U)=\rho<1$. Let $k \geq 1$ and $0 \leq \ell \leq 2^{k}-2$ be such that $b_{2^{k}+\ell+1} \leq \rho<b_{2^{k}+\ell}$ (the case $b_{2^{k+1}} \leq \rho<b_{2^{k+1}-1}$ will be considered separately). Then, because the lengths of the intervals $I_{l}^{k}$ form a nonincreasing sequence,

$$
\rho<b_{2^{k}}=\frac{\left|I_{0}^{k}\right|+\cdots+\left|I_{2^{k}-1}^{k}\right|}{2^{k}}<\left|I_{0}^{k}\right| .
$$

Then $U$ can intersect at most two consecutive intervals of step $k-1$. Hence, for all positive $t \leq 2^{k}-2$,

$$
\begin{aligned}
\mu_{h}(U) & \leq\left(\mu_{h}\left(I_{t}^{k-1}\right)+\mu_{h}\left(I_{t}^{k-1}\right)\right), & & \\
& \leq 2 h\left(b_{2^{k-1}+t}\right)+2 h\left(b_{2^{k-1}+t}\right) & & \text { by Proposition } 6 \\
& \leq 8 h\left(b_{2^{k-1}}\right) \leq 32 h\left(b_{2^{k}+\ell+1}\right) & & \text { by Lemma } 2 .
\end{aligned}
$$

Since $h$ is nondecreasing, $\mu_{h}(U) \leq 32 h\left(b_{2^{k}+\ell+1}\right) \leq 32 h(\operatorname{diam}(U))$.

Now assume $\rho$ satisfies $b_{2^{k+1}} \leq \rho<b_{2^{k+1}-1}$. Since $\rho<b_{2^{k}}$, we still have

$$
\mu_{h}(U) \leq 8 h\left(b_{2^{k-1}}\right)=8 \frac{1}{2^{k-1}}=32 \frac{1}{2^{k+1}}=32 h\left(b_{2^{k+1}}\right),
$$

and so again, $\mu_{h}(U) \leq 32 h(\operatorname{diam}(U))$. 
Therefore, if $\left\{U_{k}\right\}$ is a $\delta$-covering of $C_{a}$, we have

$$
\sum_{k} h\left(\operatorname{diam}\left(U_{k}\right)\right) \geq \frac{1}{32} \sum_{k} \mu_{h}\left(U_{k}\right) \geq \frac{1}{32} \mu_{h}\left(C_{a}\right) .
$$

Since this is true for every $\delta$-covering, we obtain

$$
\mathcal{H}_{\delta}^{h}\left(C_{a}\right) \geq \frac{1}{32} \mu_{h}\left(C_{a}\right)
$$

and therefore $\mathcal{H}^{h}\left(C_{a}\right) \geq \frac{1}{32}$.

One can also establish a certain equivalence relation among dimension functions: $h \equiv g$ if there exist constants $c_{1}$ and $c_{2}$ such that

$$
c_{1} \leq \underline{\lim }_{x \rightarrow 0+} \frac{h(x)}{g(x)} \leq \varlimsup_{x \rightarrow 0+} \frac{h(x)}{g(x)} \leq c_{2} .
$$

The following result relates the function $h$ to $\alpha(a)$.

Proposition 7. If $a \sim n^{-1 / s}$ then $h \equiv x^{s}$.

Proof. Since $a \sim n^{-1 / s}$, we have $\gamma(a)=\beta(a)=s$, and hence there exist $c>0$ and $d>0$ such that

$$
c n^{-1 / s} \leq a_{n} \leq d n^{-1 / s},
$$

and therefore

$$
C n^{-1 / s} \leq \frac{r_{n}}{n} \leq D n^{-1 / s}
$$

Hence

$$
0<c_{1} \leq \underline{\lim }_{x \rightarrow 0+} \frac{h(x)}{x^{s}} \leq \varlimsup_{x \rightarrow 0+} \frac{h(x)}{x^{s}} \leq c_{2}<+\infty .
$$

\section{References}

[BT54] A.S. Besicovitch and S.J. Taylor, On the complementary intervals of a linear closed set of zero Lebesgue measure, J. London Math. Soc., 29 (1954), 449-459, MR 0064849 (16,344d), Zbl 0056.27801.

[Bor49] É. Borel, Éléments de la Théorie des Ensembles, Albin Michel, Paris, 1949, MR 0031024 (11,88c), Zbl 0041.37502.

[CHM03] C. Cabrelli, K. Hare, and U. Molter, Some inequalities for the dimension of Cantor sets, preprint, 2003.

[CMPS03] C. Cabrelli, U. Molter, V. Paulauskas, and R. Shonkwiler, The Hausdorff dimension of p-Cantor sets, Real Analysis Exchange, to appear, 2003.

[Fal97] K.J. Falconer, Techniques in Fractal Geometry, Wiley, New York, 1997, MR 1449135 (99f:28013), Zbl 0869.28003.

[Hau19] F. Hausdorff, Dimension und äußeres Maß, Math. Ann., 79 (1919), 157-179, JFM 46.0292.01.

[Mat95] P. Mattila, Geometry of Sets and Measures in Euclidean Spaces: Fractals and Rectifiability, Cambridge Studies in Advanced Mathematics, 44, Cambridge University Press, Cambridge, 1995, MR 1333890 (96h:28006), Zbl 0819.28004. 
[Rog98] C.A. Rogers, Hausdorff Measures (second ed.), Cambridge Mathematical Library, Cambridge University Press, Cambridge, UK, 1998, MR 1692618 (2000b:28009), Zbl 0915.28002.

[Tri81] C. Tricot, Douze définitions de la densité logarithmique, C.R. Acad. Sci. Paris Sér. I Math., 293(11) (1981), 549-552, MR 0647678 (83i:28007), Zbl 0483.28011.

[Tri95] C. Tricot, Curves and Fractal Dimension, Springer-Verlag, New York, 1995, with a foreword by Michel Mendès France, translated from the 1993 French original, MR 1302173 (95i:28005), Zbl 0847.28004.

Received Received May 28, 2003. Carlos Cabrelli and Ursula Molter are partially supported by grants from the CONICET, the University of Buenos Aires and a Guggenheim fellowship. Franklin Mendivil was partially supported by NSERC.

Departamento de Matemática

Facultad de Ciencias Exactas y Naturales

Universidad de Buenos Aires

Ciudad Universitaria, Pabellón I

1428 CAPital Federal

Argentina

E-mail address: cabrelli@dm.uba.ar

Department of Mathematics and Statistics

ACADIA University

Wolfville Nova Scotia B4P 2R6

CANADA

E-mail address: franklin.mendivil@acadiau.ca

School of Mathematics

Georgia Institute of Technology

Atlanta GA 30332

E-mail address: shenk@math.gatech.edu 